\title{
El comportamiento infraestructural: prácticas relacionales en torno a la materia urbana
}

The infrastructural performance: Relational practices around urban matter

\section{Jorge Vergara Vidal* y Diego Asenjo Muñoz**}

Recibido: 15 de noviembre de 2018.

Aceptado: 13 de junio de 2019.

\section{Resumen}

Tomando en cuenta el efecto relacional que tienen los métodos con que recabamos información de lo que nos rodea, el articulo explora cómo a partir de la captura y representación datos referidos a la materialidad de las edificaciones en Chile, se dibuja un comportamiento distributivo que es también un modo de disposición diferente al que se desarrolla en torno a las tipologías arquitectónicas y a los tipos de ciudades. Los instrumentos y datos observados ayudan a ello, en la medida en que inscriben la referencia de las edificaciones sin considerar su forma o su función final, permiten la observación una cualidad propiamente infraestructural de la materia.

Palabras clave: edificación, infraestructura, materialidad, objetos de frontera, prácticas de cooperación

\begin{abstract}
Considering the relational effect of the methods with which we gather information about what surrounds us, the article explores how from the capture and representation data referring to the materiality of buildings in Chile, a distributive behavior is drawn that is also a way of different disposition to the one that develops around the architectural typologies and the types of cities. The instruments and observed data help, insofar as they record the reference of the buildings without considering their final form or function, they allow the observation of a properly infrastructural quality of the subject.
\end{abstract}

Keywords: boundary objects, construction, infrastructure, cooperation practices, materiality.

* Filiación: Escuela de Sociología, Universidad de Valparaíso, Valparaíso, Chile. Contacto: jorge.vergaravi@uv.cl

** Filiación: Corporación Municipal de Renca, Santiago, Chile. Contacto: diego.asenjo@renca.cl

Artículo de investigación científica y tecnológica producto del trabajo de investigación La gobernanza de la altura. Actores y regímenes implicados en el gobierno de las edificaciones residenciales de altura en el Gran Santiago, FONDECYT 3170016, financiado por el Comisión Nacional de Investigación Científica y Tecnológica (CONICYT)

Cómo citar: Vergara Vidal, J. y Asenjo Muñoz, D. (2019). El comportamiento infraestructural. Prácticas relacionales en torno a la materia urbana. Revista de Urbanismo, 40, 1-13. https://doi.org/10.5354/0717-5051.2018.51539 


\section{Introducción}

La materia es aquello de lo que están hechas las cosas y es, por tanto, un componente esencial de las experiencias humanas sobre el mundo. Sin embargo a pesar de su impronta sólida, sus atributos son más bien fluidos, no están fijos, sino son el resultado de sucesos procesales y relacionales que organizan sentidos y que afectan la interacción que se tiene con ella (Ingold, 2007; Law \& Mol, 1995). Esto hace posible que se hable, por ejemplo, de la imaginación o del lenguaje de la materia, que no solo son modos en los que es percibida, sino también disposiciones que permiten la colaboración entre actores con y a través de ella (Pallasmaa, 2018). El presente trabajo aborda la materia desde una perspectiva relacional que pone especial consideración en sus atributos infraestructurales, es decir, en aquellos que la presentan como un conjunto de elementos que sirve a una actividad principal, que no sólo se encuentra debajo de ésta, sino que también la soporta para que pueda desplegarse (Star y Bowker, 1995).

Para observar este modo de relación de la materia se recurrió a los datos levantados por dos instrumentos gubernamentales que, utilizados dentro de los procesos de autorización y recepción municipal de edificaciones, cuantifican los componentes materiales y técnicos de éstas. Estos instrumentos capturan información sobre un momento de la materia en que su propósito final es difuso y donde su condición no se enlaza aún con las comunidades de prácticas de la arquitectura o del urbanismo. Es un momento donde un montón de piedras - ladrillos son casi sólo eso, carentes de asociación a un proyecto singular (Hegger, Drexler y Zeumer, 2007; Zegers, 1998).

En particular los instrumentos observados, la Encuesta de Edificación y el Formulario Único de Estadísticas de Edificación (FUEE), estandarizan, cuantifican y representan la materia de una manera diferente a como lo hacen las epistemologías arquitectónicas o urbanas, como planos o como mapas (Martin, 2018), a pesar de que igualmente la asocian al proceso de edificación en las ciudades. Estos instrumentos recogen datos que al agruparlos hacen posible narrar la materia en forma de grafos o dibujos que refieren a su comportamiento en el tiempo y en el espacio, más que su forma o su función expresa.
Dentro de estas narrativas, la materia participa como un conjunto heterogéneo que constituye el soporte de la edificación, vista procesualmente. Si otros objetos epistemológicos (Ramos Zincke, 2016) o intermediadores (Vinck, 2009, 2017), como los planos o los mapas, permiten enlazar narrativamente la materia a comunidades de prácticas específicas como la arquitectura o el urbanismo, los cuadros y gráficos apelan a comunidades de prácticas diferentes, como las referidas al gobierno material, técnico, constructivo y residencial. Son útiles para reunir, por ejemplo, a los gremios constructores con los gobiernos locales y nacionales, de hecho, son instrumentos diseñados bajo esa alianza.

Considerando los anterior, el articulo explora cómo a partir de tales operaciones de captura y representación de la referencia circulante referida a la materia, se dibuja un comportamiento distributivo que es también un modo de disposición material, diferente al que se desarrolla en torno a las tipologías arquitectónicas y a los tipos de ciudades. Los instrumentos y datos observados ayudan a ello en la medida en que inscriben la referencia de las edificaciones sin considerar su forma o su función final.

El texto propone que la observación de la materialidad urbana en un momento en que su forma y su función final son aún difusos permite una consideración meramente infraestructural de ésta, acotada solo a las operaciones que habilita a partir de su agregación general. Lo anterior se expone en dos partes. En la primera se explora, utilizando las representaciones gráficas, el comportamiento del modo de distribución de la materia según la altura de las edificaciones y según su ubicación en ciudades. Esto permite apreciar un modo de agregación que coincide en el gesto con ciertas tipologías arquitectónicas y con tipos de ciudades, lo cual denota que el modo de distribución tiene un sentido habilitante respecto de las figuras tipológicas arquitectónicas y urbanas.

En la segunda parte se discute el sentido infraestructural del comportamiento anterior, partiendo de la base que estas representaciones, que no grafican comportamientos propiamente arquitectónicos ni propiamente urbanísticos, puesto que no hay obra como tal, exponen un tipo específico de relación de la materia, que carece de un propósito manifiesto y que se expresa en formas o agregaciones difusas. 
La metodología empleada, como se mencionó, se basa en la sistematización y análisis estadístico de los datos sobre material elaborado utilizado en edificaciones de obra nueva, recogidas por la Encuesta de Edificación, aplicada entre 1990 y 2001, y del Formulario Único de Estadísticas de Edificación (FUEE), aplicado desde el 2002 en adelante, ambos por el Instituto Nacional de Estadísticas (INE).

\section{El comportamiento infraestructural}

Pese a su condición sólida, los atributos de la materia son resultado de entramados de procesos y relaciones en que ésta era involucrada (Ingold, 2007; Law \& Mol, 1995). Salvo en su estado primigenio, en que la (mal) suponemos aislada, la materia se encuentra siempre en diversos estados de disposición física y simbólica respecto de diferentes comunidades de prácticas humanas que marcan, a través de ello, sus modos de relación y gobierno con lo material. Las diferencias que se establecen entre materia prima, bruta y elaborada son parte de esto, así como los modos en que las comunidades de prácticas integran en éstas y en sus valores tales estados de disposición.

En la escultura, por ejemplo, tanto el estado de elaboración como la genealogía del material son extremadamente relevantes (Gazitúa, 1988). Sus miembros son capaces de denotar de forma diferente materias como la madera y el acero, y a la vez, distinguir en ello la trayectoria que separa al árbol de la tabla de madera, y la que recorre el mineral para convertirse en varilla de acero. Para la arquitectura, en cambio, estas trayectorias materiales no resultan tan centrales, o al menos no lo son en comparación con el lugar que ocupa en sus prácticas la poética de la composición formal. Dentro de esta comunidad, la diferencia entre material elaborado y material bruto resulta menos, ambos son primarios y ambos pueden constituir el punto de apoyo matérico de la forma construida (Pérez de Arce, 1988; Zegers, 1988).

Por su parte, para quienes planifican y ordenan ciudades, el estado de elaboración de los materiales que se integran a ellas resulta meramente anecdótico, puesto que los llenos y vacíos dentro de un plano escasamente discuten las composiciones materiales integradas a esa diferenciación. El urbanismo, propiamente tal, tampoco se pronuncia sobre los orígenes de los materiales con que sus obras son realizadas, dejando ello al mercado de materiales, que está fuera de su ámbito de acción. Esto puede ser extendido al conjunto de la reflexión sobre la escala urbana. La ciudad genérica de Koolhaas es un buen ejemplo de esto, en ella la materia constructiva carece de notación, y cuando la ciudad pasa de la horizontalidad a la verticalidad, el mismo Koolhaas considera que quienes tienen menos dinero habitan la tierra y quienes tienen más habitan el aire, tornando invisibles los soportes matéricos (2014), "la forma tiene voz, mientras la materia permanece silenciosa o ausente" (Pallasmaa, 2018, p. 45).

Estas diferencias entre comunidades de prácticas que pueden ser consideradas como cercanas, obedecen al diverso valor que adquiere dentro de ellas la relación entre forma y propósito, y el papel que se le asigna a la materia dentro de cada sistema de sentidos. No hay una sola fórmula para esto. Habrá quien, como Cazú Zegers (1988) proponga una ética material alojada intrínsecamente en la forma construida y quien, como Rem Koolhaas (2014), le supedite completa e invisiblemente a la solución de la forma. Pero en general, tanto para la arquitectura como para el urbanismo, el propósito de la obra será mejor explicado por la forma que por la composición material, tanto en el caso de la obra singular (Baudrillard y Nouvel, 2003), como en el caso de sus obras masivas, donde los propósitos se expresan como tipologías (Muszbek y Froimovich, 2018). No hay aquí una desconsideración de la materia dentro de estas comunidades de prácticas sino el uso, por parte de ellas, de una fórmula de interacción específica entre la materia y la forma (Pérez, Aravena y Quintanilla, 2007).

En el terreno de la arquitectura, sostienen Pérez, Aravena y Quintanilla, la interacción entre forma y materia tiene un carácter dialéctico, no se imponen una a la otra, ni se derivan entre sí mecánicamente. Dentro de este esquema relacional, los componentes materiales de una obra configuran una dimensión tectónica, donde se hacen evidentes su esbeltez, su compacidad, su blandura, su ligereza y su transparencia, es decir, sus condiciones táctiles, auditivas y olfativas, puestas en función de un propósito (Deplazés, 2010; Pérez et al., 2007).

La materia aparece entonces no solo como un componente esencial de las cosas, sino también como un aspecto relacional mediante el cual éstas son posibles de 
enlazar a prácticas y a colectivos políticos mayores. Como sostienen Penny Harvey y Hannah Knox (2012), todo lo que sabemos de la materia cambia con el tiempo y ello no es porque en sí la materia y sus composiciones cambien, sino porque las significamos de modos diferentes constantemente, porque sus historias cambian, porque sus asociaciones son heterogéneas.

La materia considerada relacionalmente no produce distinción entre lo material y lo ideal, pero ello no significa que no genere diferencias (Law y Mol, 1995). Estas diferencias son constituidas y organizadas mediante estrategias semióticas, como ocurre con los mapas, los planos y los formularios, las cuales juegan un rol importante en los modos de ordenamiento del mundo. Los modos como es representada la materia en las gráficas anteriormente vistas nos proponen modelos para ordenar el mundo que observamos, tanto en la escala de las edificaciones como en la escala de las ciudades, que solo es posible de articular dentro de un marco de interacciones que no es el de las obras arquitectónicas o urbanas, sino dentro de un hecho infraestructural. Esas formas de orden no existen fuera de ese marco de interacciones (Ingold, 2007)

A partir de esta dimensión relacional de la materialidad, cabe discutir sobre los efectos epistemológicos que esto puede tener sobre la forma de entender y gobernar los hechos de arquitectura y los hechos urbanos. Como se ha visto, en general, estos han sido explicados y caracterizados a partir de la singularidad (Borchers, 1968), de manera lo que se obtiene de ello se acota al análisis de sus tendencias estéticas o funcionales. El resultado es diferente si se abordan desde una perspectiva infraestructural, pues allí el análisis involucra la relacionalidad de todo un colectivo, que no es igual a la suma de sus partes.

Jane Bennett (2010) sostiene que la costumbre epistemológica de concebir la materia como algo intrínsecamente inanimado puede ser uno de los obstáculos más fieros para el surgimiento de modos sostenibles de gestión de nuestros entornos vitales. A cambio, propone considerarla como una serie de conjuntos vibrantes y complejos, que interactúan constantemente entre sí en formas determinadas y bajo marcos relacionales en los cuales es reconocida su capacidad de agencia política. Observada de esta manera, las obras de arquitectura y las obras urbanas pueden ser desplazados hacia otros colectivos materiales organizados, esta vez, en torno a la materia, su composición, utilidad u otras alternativas de agencias comunes que se constituyan como otras posibilidades políticas.

En el caso observado, la agencia común es constituida en torno a los instrumentos Encuesta y FUEE. Estos funcionan como objetos ubicados en las fronteras entre comunidades de prácticas diferentes, capaces de enlazarlas por medio de la perspectiva infraestructural que proponen (Star, 1999, 2002). Esta posibilidad política no es casual, está considerada en el diseño y la implementación de los mismos instrumentos y en el rol que tuvieron en ello las diversas entidades gubernamentales y privadas involucradas.

Los dos instrumentos fueron diseñados de forma consecutiva por el Instituto Nacional de Estadísticas y por el Ministerio de Vivienda, a través de sus direcciones técnicas, que eran y son las encargadas de la elaboración procesos de los estándares técnicos aplicables a las materialidades constructivas. La Encuesta de Edificación se diseñó durante toda la década de 1980, tanto en lo que refiere a sus métricas como a sus modos de implementación, lo que involucró también a diversos municipios que pre-testearon el instrumento. Sin embargo, cuando ésta comenzó a ser aplicada a nivel nacional algunas de las formas de medir la materia se encontraban obsoletas respecto su entorno socio técnico. Algunas materialidades fueron prohibidas, como ocurrió con los asbestos, se habían introducido al mercado materias no contempladas en el instrumento, o la plataforma tecnológica de registro (computadores, web) había evolucionado. Todo esto llevó a la necesidad de reformular el cuestionario con el fin de mejorar la calidad de su información.

EI FUEE, que reemplazó a la Encuesta, fue elaborado en conjunto con la Cámara Chilena de la Construcción, entidad privada que agrupa a las empresas y asociaciones que participan en los procesos edificatorios, y que cuenta con empresarios, técnicos, académicos y expertos, que a su vez integran las diversas comisiones del MINVU que elaboran las diversas normas técnicas materiales. Así, el FUEE y sus métricas, reúne en su proceso de diseño y aplicaciones a comunidades de prácticas 
gubernamentales, constructivas y comerciales que se relacionan con la materia de maneras distintas a la arquitectura y el urbanismo. Tales diferencias relacionales quedan inscritas en la epistemología de los instrumentos, en el modo como estos recogen y traducen la referencia de la materia de la que deben dar cuenta.

Debido a ello, los instrumentos observados y las representaciones de sus datos permiten apreciar un comportamiento de la materia que no está definido por la obra, su forma o su función expresa, sino por aquello a lo que sirve o a lo que sustenta, en este caso, por el proceso de edificación en las ciudades chilenas.

Este comportamiento puede ser calificado de infraestructural si se considera lo propuesto por Star y Bowker (1995) en orden tanto a que toda infraestructura expresa un sentido de soporte para el despliegue de una actividad principal (edificación, en este caso), como que este emerge de necesidades de información y trabajo, configuradas en situaciones cooperativas, que son necesarias para que actores y comunidades de prácticas diferentes hagan cosas juntos (Star y Greisemer, 1989), como por ejemplo, sucede con los gremios constructores y los gobiernos locales y nacional. De esta manera, la condición infraestructural de la materia emerge configurada por las necesidades de información de comunidades de prácticas diferentes que la requieren para poder cooperar entre sí.

Entidades gubernamentales (INE, MINVU, municipios), privadas y gremiales (Cámara Chilena de la Construcción), que participan en el diseño y aplicación de los instrumentos, buscan articular un enlace que les permita conversar y cooperar entre sí. La evaluación del comportamiento de la materia, de sus ritmos y tendencias mediante cuadros y gráficos, colabora con ello, así como la asignación a ésta de un papel infraestructural. La materia sólo adquiere tal comportamiento infraestructural en la medida que es llevada a ello, por los entramados relacionales en los que es incluida o participa.

\section{El comportamiento distributivo}

Bosquejar el comportamiento agregativo de la materia utilizada en las edificaciones chilenas durante un periodo de veintiséis años requirió, en primer lugar, organizar una imagen continua con dos contabilidades diferentes, la que provenía de la Encuesta de Edificación, aplicada hasta el año 2001, y la que correspondía al Formulario Único de Estadísticas de Edificación (FUEE), aplicado desde el 2002 en adelante.

Cada uno de estos instrumentos había sido elaborado en un momento socio-material particular, por tanto las métricas mediante las cuales clasificaban y estandarizaban estaban situadas en la configuración sociotécnica de cada periodo, lo cual afectaba los posibles cálculos sobre distribución a realizar a partir de ellos. EI caso del asbesto, entre otros, ejemplifica bien lo que ocurre entre un instrumento y otro.

Las placas de asbesto eran contabilizadas por la Encuesta de Edificación, por lo cual es posible saber que su uso era corriente y estable durante la década 1990, a pesar de que Chile había firmado en 1983 un acuerdo para restringir su uso. Fue prohibido en Chile, oficialmente, en el año 2001, mismo año en que el FUEE está en su proceso final de diseño e implementación. De manera que a partir de la implementación de este segundo instrumento el asbesto desaparece de la contabilidad material.

Otro caso es el del ladrillo. En la Encuesta de Edificación, el ladrillo es una sola materialidad, en el FUEE, corresponde a dos cuya diferencia es el grado de elaboración: ladrillo de fabricación industrial y el de fabricación artesanal. Y otro caso, entre otros, son las materias utilizadas en puertas y ventanas, el FUEE las cuenta, la Encuesta no, de manera que el FUEE entrega una imagen más heterogénea que la que es posible organizar a partir de los datos de la Encuesta.

De manera que, para bosquejar una imagen continua, hubo que enlazar los dos instrumentos y, con ello, sus momentos sociomateriales y sus normas particulares de agrupación, los estándares contenidos en cada uno de ellos. Para ello se homologaron categorías y se organizaron en un canon material continuo, que reconoce las materialidades de adobe, bloque cemento, hormigón, ladrillo, madera, metal (panel preformado y muro cortina), piedra y otras combinaciones (como ferrocemento, metal vidrio, entre otros); y se organizaron en un canon espacial continuo, es decir, se consideraron solo las materialidades utilizadas en muros y cerramientos, ya que era la ubicación que los dos instrumentos tenían en común.

Los datos dibujan un proceso de progresivo consumo de materia, cada año hay incremento respecto del 
anterior. El registro de su distribución según la altura de las edificaciones está acotado al rango que va entre uno y nueve pisos o más, por tanto la información que entrega respecto a este aspecto de la forma de estas es bastante difusa. Sin embargo, como es posible apreciar en la Figura 1, este aspecto igual puede ser representado si se organiza la distribución de las materialidades según rango de altura, en este caso, el conjunto de las materialidades utilizadas en muros y cerramientos en el periodo observado en el conjunto del país.

La representación que expresa la Figura 1 permite apreciar el gran volumen de materialidad utilizada en las edificaciones de uno a tres pisos de altura, lo cual es seguido, incluso en los altos y bajos de la evolución, por el volumen de materiales utilizados en edificaciones de nueve y más pisos, que superan al volumen utilizado en edificaciones de altura intermedia. El $71,6 \%$ de las materialidades fueron utilizadas en edificaciones de uno a tres pisos, mientras el 7,7\% fue utilizada en edificaciones de entre cuatro a cinco pisos. Esto indica una fuerte inversión material, el 79,3\% del conjunto utilizado, a la construcción en baja altura en el periodo observado, lo cual conlleva una expansión del área construida y un agotamiento del suelo urbano (Hidalgo, 2007).

A contrapelo de esta tendencia predominante se puede reconocer una creciente verticalización de las edificaciones (Vergara Vidal, 2017). Un 20,7\% de las materialidades utilizadas en el periodo observado se ocupó en edificaciones de más de seis pisos de altura y en particular un $16,7 \%$ se ocupó en edificios de más de nueve pisos de altura, demostrando una tendencia consistente en los último diez años.

Si bien, la Figura 1 no representa tipologías arquitectónicas, es claro que las edificaciones de entre uno a tres pisos corresponden a casas, departamentos en bloques de vivienda sociales o a edificaciones comerciales pequeñas. Un análisis similar, realizado solo con los datos de la Región Metropolitana de Santiago (Vergara, 2018), indicó que en ese rango de altura un $45 \%$ de las edificaciones tiene un uso no residencial, y no hay motivos para suponer que la tendencia nacional sea distinta, dado el alto peso del Santiago Metropolitano en el agregado del país, como se verá más adelante.

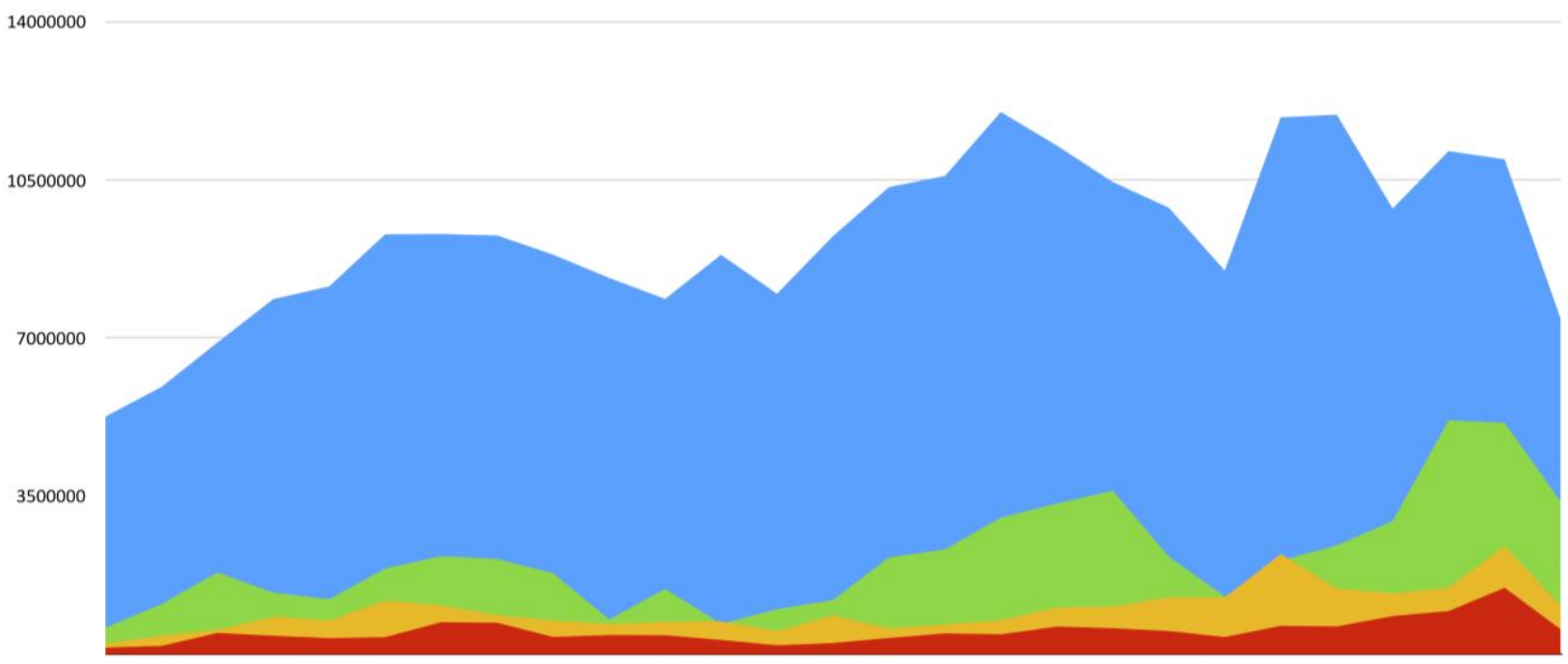

199019911992199319941995199619971998199920002001200220032004200520062007200820092010201120122013201420152016

Figura 1. $\mathrm{m}^{2}$ de materialidades utilizadas en muros de edificaciones en Chile según altura y año (1990-2016). Fuente: INE, Encuesta de Edificación (19902001) y Formulario Unificado de Estadísticas de Edificación (2002-2016). 
Asimismo, el consumo de material destinado a edificaciones de más de seis pisos de altura $(20,7 \%)$ no parece estar asociado a otra tipología que a edificios de altura, aunque claramente esto no dice nada concreto acerca de las múltiples disposiciones que las formas tipológicas pueden adquirir (Muszbek y Froimovich, 2018). Sin embargo, en este caso la tendencia de ocupación tiene un $80 \%$ de destino residencial (Vergara Vidal, 2017) y la representación de la Figura 1 muestra además que el incremento de consumo material en ese tipo de edificaciones aumenta progresivamente, lo que da cuenta de una adopción paulatina, pero tendencial, de los edificios de altura como infraestructura residencial.

Si se considera un trazo menos grueso para mostrar la distribución de la materialidad en las edificaciones según los pisos de altura, considerados en la medición de los instrumentos observados (Figura 2), es posible notar que los agregados de edificaciones de menor altura presentan una mayor heterogeneidad material, lo cual constituye un segundo gesto tipológico que, sin pronunciarse sobre la forma en sí, muestra cómo se comporta la materia que la soporta.

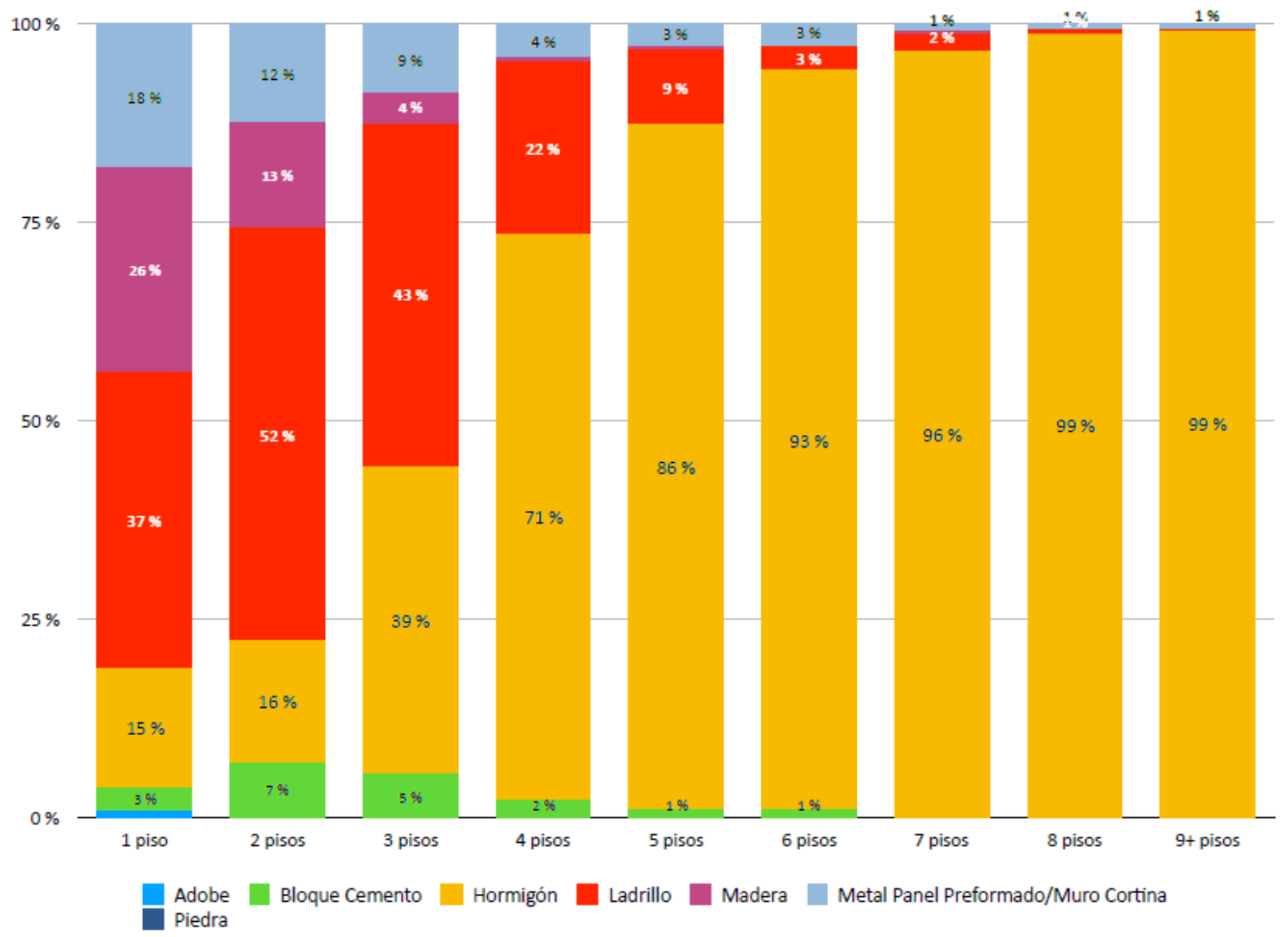

Figura 2. Porcentaje de materialidades utilizadas en muros de edificaciones de Chile según altura y año (1990-2016). Fuente: INE, Encuesta de Edificación (1990-2001) y Formulario Unificado de Estadísticas de Edificación (2002-2016). 
La Figura 2 hace posible apreciar como los instrumentos capturan la narrativa material del comportamiento tipológico (Argan, 1983; Moneo, 1978). Indican que las formas de baja altura integran más diversidad material que las de altura mayor, haciendo evidente el carácter infraestructural de la materia respecto a la forma, como ocurre claramente con la presencia del hormigón en los edificios sobre los seis pisos de altura y la progresiva ausencia del ladrillo en los mismos casos.

El comportamiento de las composiciones materiales según las altura de las edificaciones puede indicar un tipo de estandarización mayor en las edificaciones sobre los seis pisos de altura, dado que los porcentajes de las materialidades incluidas en dichas edificaciones son cada más similares según se progresa en la altura (Figura 2), indicando la posibilidad de un ejercicio de repetición a nivel sus composiciones materiales. Esta estandarización no afectaría necesariamente a la forma arquitectónica, sino a las prácticas materiales que las soportan.

En el mismo sentido, la representación de la distribución material expresada en la Figura 2, muestra que en el caso de las edificaciones de menor altura, esta estandarización debiese ser mucho menor, dada la heterogeneidad observada en sus composiciones materiales o, por lo menos ir por otro lado, el de la singularidad compositiva. Esto es observable no solo en las diferencias entre las propuestas arquitectónicas de las casas o de las viviendas de baja altura, sino también dentro de sus mismos conjuntos que, en el proyecto se presentan como formas estandarizadas, pero cuya formas y composiciones son alteradas en el tiempo por sus dueños o sus usuarios hasta tornarlas diferentes unas de otras (García-Huidobro, Torres y Tugas, 2008; Martín, 2015).
Con todo, una estandarización a partir de las prácticas materiales es más clara de suponer al observar el caso la homogénea composición de las edificaciones de alturas mayores, que la heterogeneidad de las menores. Al utilizar menos materiales en su obra gruesa, las normas técnicas que rigen las composiciones de las edificaciones sobre los seis pisos son menores en número, y los actores responsables de su construcción están más especializados que los pueden construir o intervenir una edificación de baja altura.

Sin embargo, los materiales utilizados en las edificaciones de baja altura son igualmente estandarizados. Ni el metal panel, ni el ladrillo o la madera son producidos carentes de estándares y eso es recogido por los instrumentos en el modo de inscripción de los datos. La diferencia está en lo estandarizado de las prácticas de construcción que la composición lleva de la mano y, ya relacionado con los aspectos programáticos de las formas, de las prácticas de habitar que están asociadas a ello. En ese marco, la homogeneidad compositiva de las edificaciones sobre los seis pisos de altura ayuda a considerar una relación infraestructural entre la materia y la forma de las edificaciones en la cual el comportamiento de las composiciones puede funcionar como un indicador de la presencia de formas o tipologías constructivas y de su distribución en las ciudades del país (Pérez López, 2015).

Dado que las composición material de estas edificaciones es similar, se sumó el total de $\mathrm{m}^{2}$ usados en muros de edificación nueva y se verificó su distribución primero en las tres ciudades metropolitanas del país (Figura 3) y luego en algunas de las ciudades intermedias del mismo (Figura 4). El resultado indica que el material elaborado destinado a este tipo de edificaciones se comporta de una manera concentrada territorialmente. 


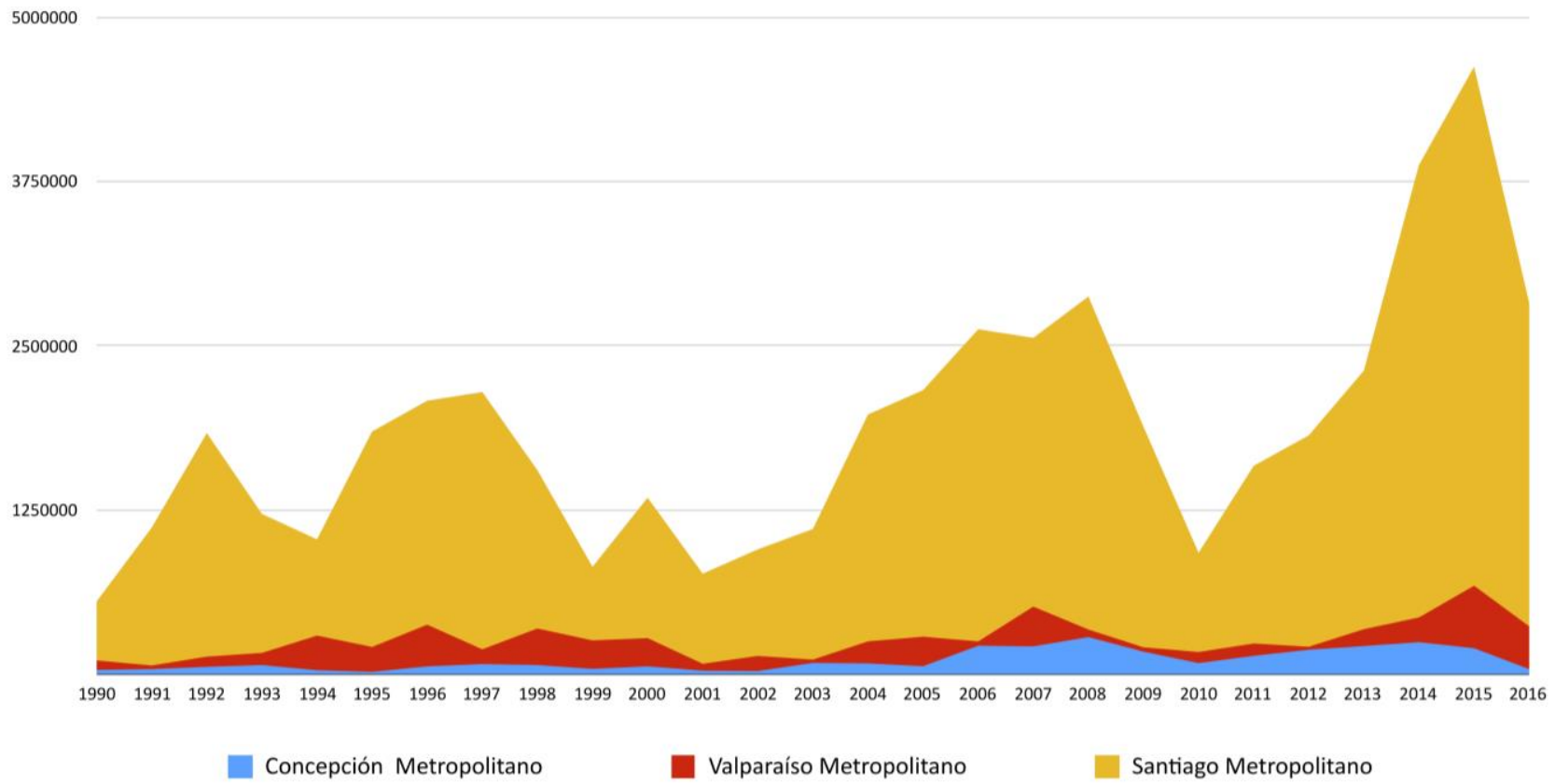

Figura $3 . \mathrm{m}^{2}$ de materialidades utilizadas en muros de edificaciones de 6 pisos y más de obra nueva en ciudades metropolitanas de Chile (1990-2016). Fuente: INE, Encuesta de Edificación (1990-2001) y Formulario Unificado de Estadísticas de Edificación (2002-2016).

Cabe indicar que las ciudades metropolitanas chilenas no sólo son las más pobladas, sino también están compuestas por conjuntos de comunas, que corresponden a las unidades político-administrativas mínimas del país (Arenas, Hidalgo y Aliaga, 2009). Política y administrativamente las ciudades metropolitanas no existen, no tienen límites que las fijen como tales ni eligen autoridades. Pero sí existen materialmente, en tanto continuo construido, lo que posibilita un bosquejo tentativo. En pos de ello el INE (2007), mismo organismo responsable de las encuestas observadas, propuso una forma de agregación de comunas y localidades que daban como resultado las tres ciudades metropolitanas.

Para el caso de agregar al Santiago Metropolitano, se considera al conjunto de las localidades urbanas de la Provincia de Santiago; las localidades urbanas de las comunas de Puente Alto, San Bernardo, Peñaflor, P. Hurtado y Pirque; y las localidades de Alto Jahuel, Buin y Viluco (en la Comuna de Buin), Bajos de San Agustín (en la comuna de Calera de Tango), Colina (comuna de Colina), Batuco, Estación Colina y Lampa (Comuna de Lampa). Por su parte, el Valparaíso Metropolitano incluye las comunas de Concón, Quilpué, Villa Alemana y Viña del Mar, y las localidades de Placilla de Peñuelas y Valparaíso de la
Comuna de Valparaíso. Mientras, el Concepción Metropolitano incluye las localidades urbanas de las comunas de Concepción, Chiguayante, Hualpén, Penco, Talcahuano, San Pedro de la Paz, Tomé, Coronel y Lota (INE, 2007).

La representación del comportamiento de las materialidades, en edificaciones sobre los seis pisos de altura, en estos tres conjuntos urbanos expresa no sólo un creciente consumo material que puede traducirse como una mayor construcción de este tipo de edificaciones en estas ciudades, sino además su alta concentración en el Santiago Metropolitano (Figura 3). De hecho, en términos del periodo de tiempo considerado, el Santiago Metropolitano captura el $69,9 \%$ de la materialidad destinada a edificaciones sobre los seis pisos de altura, mientras el Valparaíso Metropolitano captura solo el 9,7\% y el Concepción Metropolitano, captura el 3,9\%.

Los tres conjuntos metropolitanos capturan el $83,5 \%$ de la materialidad invertida en edificaciones sobre los seis pisos, lo que podría indicar una posible relación entre el tamaño de las ciudades y la densificación de sus centros y pericentros mediante tipologías de altura. En tal sentido es interesante que los aumentos de material dedicado a ellas, en 1995-1998, en 2004-2008 y en 2013-2015, se 
producen en los tres casos y que algo similar ocurre en algunas de las ciudades que capturan el $16,5 \%$ restante de la materialidad puesta en estas edificaciones.

De estas, hay seis ciudades que explican el $10,3 \%$ de las materialidades empleadas en edificaciones sobre los seis pisos de altura, las cuales no muestran comportamientos necesariamente similares entre sí, aunque hay gestos comunes. La representación de los datos expuesta en la Figura 4, por ejemplo, muestra que Antofagasta, Iquique-Alto Hospicio y La Serena- Coquimbo tienen incrementos de consumo material similares a los vistos en las ciudades metropolitana. Son también las que capturan mayor volumen material y las de mayor crecimiento poblacional.

La ciudad de Temuco-Padre Las Casas, por su parte, explica el $1,3 \%$ de los consumos materiales en edificaciones en altura del país y que parece seguir un ritmo de consumo a contrapelo de los antes vistos; mientras que Algarrobo es la única de estas ciudades que no corresponde a una ciudad intermedia, sino a un expansión de segundas viviendas costeras del Santiago Metropolitano. De hecho, su población estable no supera los 10 mil habitantes y supera en consumo material de edificaciones de altura a ciudades con poblaciones diez veces mayores, como Copiapó-Tierra Amarilla o Rancagua.

Con todo, cabe considerar que, en el periodo considerado y 291 ciudades, las tres metropolitanas, las cinco intermedias y la ciudad costera recién señaladas explican en conjunto el $93,8 \%$ del total de materialidades utilizadas en las edificaciones de altura observadas, es decir que casi explican el conjunto del fenómeno. De las ciudades que quedan fuera de estas nueve, ninguna supera el $1 \%$, aun cuando ochenta y ocho presentaron consumos materiales para edificación en altura, lo que indica que, sin ser irrelevantes $y$ habiendo crecido poblacionalmente también en el periodo observado, se han densificado por medio de otros modos infraestructurales.

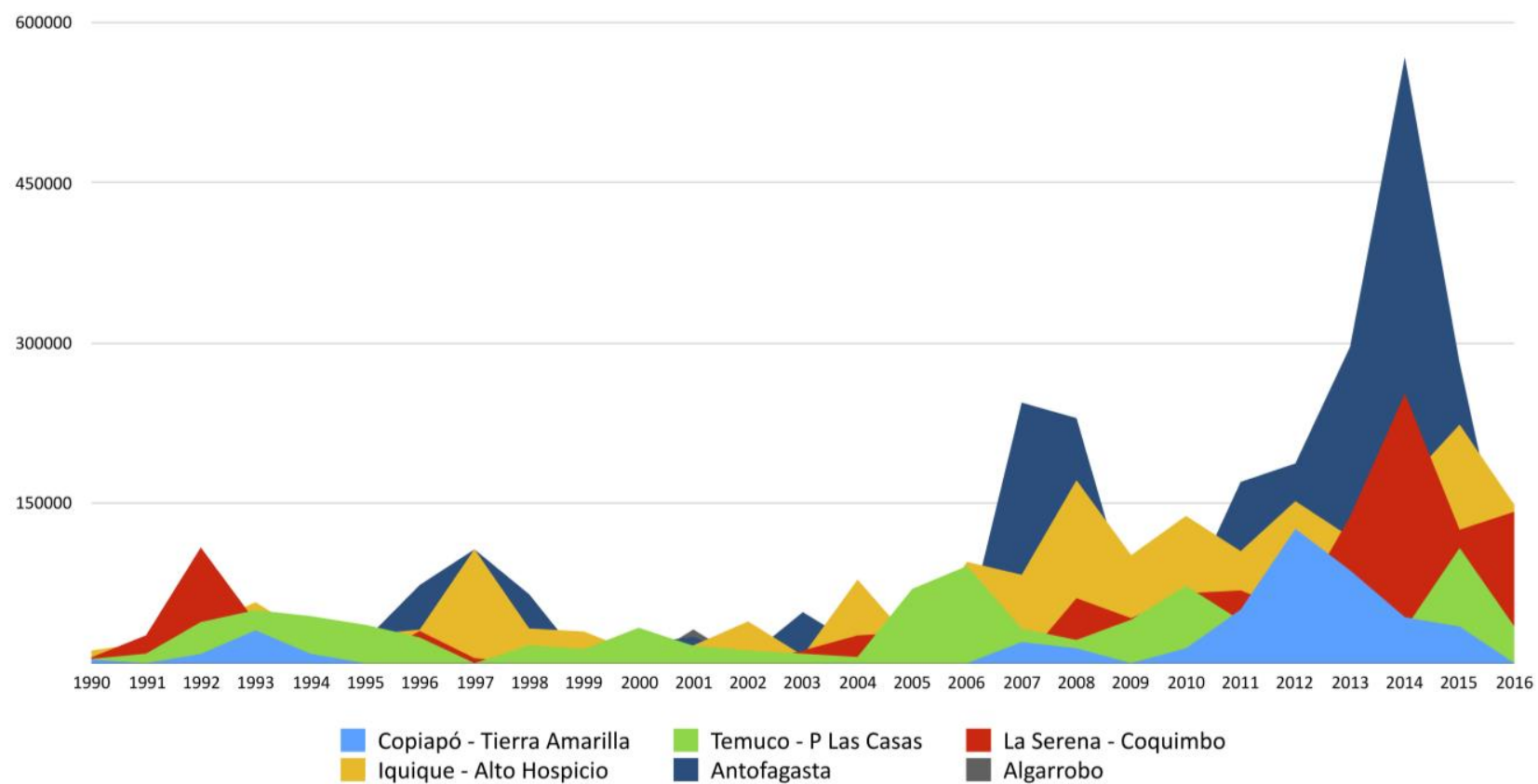

Figura 4. $\mathrm{m}^{2}$ de materialidades utilizadas en muros de edificaciones de 6 pisos y más de obra nueva en ciudades intermedias de Chile (1990-2016). Fuente: INE, Encuesta de Edificación (1990-2001) y Formulario Unificado de Estadísticas de Edificación (2002-2016). 
El proceso constructivo, visto a través de sus materialidades, evidencia intensidades y ritmos diferentes según la escala y la entidad de observación. La imagen que se construye del conjunto nacional difiere de la que se obtiene al observar las escalas locales. Las ciudades resultan puntos de observación claves (Sassen, 2005). Las ciudades metropolitanas, intermedias y pequeñas muestran rasgos de estrategias materiales diferentes, y eso no solo habla de diversidad de sus industrias, formas y composiciones materiales, sino también de requerimientos diferentes a su gobernanza urbana. Las ciudades metropolitanas, por ejemplo, pendulan entre la expansión de sus áreas y la densificación de sus centros, entre modalidades de transporte extendidos y otros de cercanías, sin lograr establecer entre esto una dualidad armónica o una hegemonía clara. Lo observado indica que algunas ciudades intermedias también avanzan hacia allá, con el mismo agravante de perder, en su crecimiento, la coincidencia entre su área construida y su unidad administrativa (Arenas et al. 2009).

\section{Conclusiones}

En lo anterior, se ha observado como el análisis de los datos y representaciones, provenientes de la aplicación consecutiva de dos instrumentos utilizados para su autorización y recepción municipal de las edificaciones, permiten apreciar en sus componentes materiales un atributo infraestructural.

Este es efecto tanto del momento en que los instrumentos capturan la información sobre las materialidades de las edificaciones, como de las necesidades información respecto de ellas provenientes de las diferentes comunidades de prácticas que estuvieron involucradas en sus diseños.

En lo que refiere a lo primero, como se va visto los instrumentos capturan a la materia en un momento en el que su propósito final es difuso, donde no es posible atribuir a ella una disposición arquitectónica o una ubicación dentro del ordenamiento urbano, sino sólo, y específicamente, verificar sus modos de distribución dentro de lo urbano. Al respecto destacan dos gestos, el de su distribución según la altura de las edificaciones, que permite ver la sombra de las tipologías arquitectónicas, y el de su distribución en las ciudades chilenas, que parece moverse siguiendo a la población, y que permite ubicar el fenómeno de la verticalización de las edificaciones en las ciudades metropolitanas y en aquellas intermedias con rápido crecimiento.

Es a través de esto que los cuadros y gráficos, que dan cuenta de los datos levantados por los instrumentos en cuestión, narran un modo relacional particular de la materia, donde ésta no es enlazable ni por las narrativas propias de la arquitectura ni por las del urbanismo pues no constituye forma ni ordenamiento. Se ha propuesto considerar dicho comportamiento como infraestructural debido a que los modos de distribución observados enuncian tanto formas en que la materia se configura para ser soporte de otros fenómenos, como necesidades de información que comunidades de prácticas diferentes requieren para la cooperación entre sí.

Narrada como infraestructura, la materia aún puede performar ademanes de forma y ordenamiento, como sucede con su distribución según la altura de las edificaciones y según las ciudades, pero ello no es todo. Puesta la condición infraestructural delante de otras posibles formas relacionales (Star y Bowker, 1995), queda en evidencia el efecto de la composición material en la configuración de soporte de las formas enunciadas y en la cultura material del país y de sus ciudades.

Propuesta como un orden práctico por medio de su condición infraestructural, la materia y sus modos de distribución pueden ser recurridos como justificación de acciones privadas y públicas. Como sucede cuando, los gremios constructores argumentan déficits en las viviendas, los espacios o las obras públicas. En tales demandas no hay una justificación propiamente arquitectónica (forma) o urbanística (orden), sino de distribución del gasto público hacia un motivo infraestructural.

Estas posibilidades narrativas, que emergen de lo que se ha indicado en estas líneas como comportamiento infraestructural de la materia, debieran estar presentes no solo en objetos epistémicos como los instrumentos observados, sino en el conjunto de los objetos constituidos, en las fronteras entre las comunidades de prácticas ya mencionadas, y dispuestos en función de habilitar la cooperación entre ellas.

El número y disposición de estos objetos entregaría una interesante perspectiva de las prácticas de cooperación en torno a la materia que es, en definitiva, lo 
que articula aquello que se ha identificado aquí como comportamiento, no otra cosa que un tipo particular de relaciones que bajo ocupan el signo de la infraestructura para describir un modo específico de cooperación socio material (Star y Ruhleder, 1996).

A partir de lo observado y expuesto anteriormente, cabe concluir que lo infraestructural surge como un sistema de valores bajo cuyo signos se da la cooperación entre comunidades de prácticas diferentes, y el comportamiento de la materia bajo esa designación corresponde al modo en que esta es integrada a un tipo de relaciones específicas que no tiene que ver ni con la forma ni con el ordenamiento en el plano. Explorar esta perspectiva respecto de la materia utilizada en edificaciones y ciudades resulta interesante pues permite inquirir, más allá de las variaciones en sus composiciones y consumos, las comunidades y modos de orden a los que sirve [R]

\section{Referencias}

Arenas, F., Hidalgo, R. y Aliaga, G. (2009). Ciudades medias en la macrozona central de Chile transformaciones socioespaciales en un contexto de metropolización. En, R. Hidalgo, C. de Mattos y F. Arenas (Eds.), Chile: Del país urbano al país metropolitano (pp. 303-321). Santiago: Editorial Pontificia Universidad Católica de Chile.

Argan, G. C. (1984). Tipología. Summarios, (79), 2-14.

Baudrillard, J. y Nouvel, J. (2003). Los Objetos Singulares: Arquitectura y Filosofía. Buenos Aires: Fondo de Cultura Económica

Borchers, J. (1968). La Institución Arquitectónica. Santiago de Chile: Editorial Andrés Bello.

Bennett, J. (2010). Vibrant Matter. A Political Ecology of Things. Durham: Duke University.

Deplazés, A. (Ed.) (2010). Construir la arquitectura. Del material en bruto al edificio. Un manual. Barcelona: Gustavo Gili

García-Huidobro, F., Torres, D. y Tugas, N. (2008). iEI tiempo construye! Time builds! Barcelona: Editorial Gustavo Gili.

Gazitúa, F. (1988). Materia. Arq (39), 18-19. Disponible en http://www.edicionesarq.cl/1998/arq-39-materia/

Harvey, P. \& Knox, H. (2012). The Enchantments of Infrastructure. Mobilities, 7(4), 521-536. https://dx.doi.org/10.1080/17450101.2012.718935

Hegger, M. Drexler, H. y Zeumer, M. (2010). Materiales. Barcelona: Gustavo Gili
Hidalgo, R. (2007). ¿Se acabó el suelo en la gran ciudad?: Las nuevas periferias metropolitanas de la vivienda social en Santiago de Chile. EURE, 33(98), 57-75. https://dx.doi.org/10.4067/S0250$\underline{71612007000100004}$

Ingold, Tim (2007). Materials against materiality. Archaeological Dialogues, (14), 1-16 https://dx.doi.org/10.1017/S1380203807002127

Instituto Nacional de Estadística (2007). Definición y Listado de Ciudades de Chile. Disponible en http://www.observatoriourbano.cl/Docs/index.asp.

Koolhaas, R. (2014). La ciudad genérica. Acerca de la Ciudad. Barcelona: Gustavo Gili

Law, J. \& Mol, A. (1995). Notes on Materiality and Sociality. The Sociological Review, 43(2), 274-294. https://doi.org/10.1111/i.1467-954X.1995.tb00604.x

Martín Blas, S. (2015). ¿El Tiempo construye la Arquitectura?: Construcción y autoconstrucción en la arquitectura de vivienda social. A\&P Continuidad, (3), 80-89.

Martin, R. (2018). On Infrastructure. Reinhold Martin interviewed by Pedro Correa and José Lemaitre. $A R Q$ (99), 12-27. https://dx.doi.org/10.4067/S0717$\underline{69962018000200012}$

Muszbek, J. y Froimovich, J. (2018). ¿Cómo vivimos? Taller de vivienda - Workshop Santiago. ARQ, (98), 120-127. https://dx.doi.org/10.4067/S0717$\underline{69962018000100120}$ 
Moneo, R. (1978). On typology. Oppositions, (13), 22-44. Disponible

en https://doarch152spring2015.files.wordpress.com/20 15/01/moneo on-typology oppositions.pdf

Pallasmaa, J. (2018). Esencias. Barcelona: Gustavo Gili

Pérez, F., Aravena, A. y Quintanilla, J. (2007). Los hechos de arquitectura. Santiago de Chile: ARQ Ediciones

Pérez de Arce, R. (1988). De carne y hueso. Arq (39), 2-7. Disponible en http://www.edicionesarq.cl/1998/arq39-materia/

Pérez López, I. (2015). Hacia una arquitectura y un urbanismo infraestructural. Urbano, 18(32), 26-37. http://revistas.ubiobio.cl/index.php/RU/article/view/ 1974

Ramos Zincke, C. (2016). La producción de la pobreza como objeto de gobierno. Santiago de Chile: Ediciones Universidad Alberto Hurtado

Star, S.L. (1999). The Ethnography of Infrastructure. En, American Behavioral Scientist, 43(3), 377- 391. http://dx.doi.org/10.1177/00027649921955326

Star, S.L. (2002). Infrastructure and ethnographic practice: Working on the fringes. Scandinavian Journal of Information Systems, 14(2), 107-122. Disponible en http://aisel.aisnet.org/sjis/vol14/iss2/6

Star, S.L. y Bowker G.C. (1995). Work and infrastructure. Communications of the ACM, 38(9), 41. https://dx.doi.org/10.1145/223248.278461

Star, S.L. \& Griesemer, J.R. (1989). Institutional Ecology, 'Translations' and Boundary Objects: Amateurs and Professionals in Berkeley's Museum of Vertebrate Zoology, 1907-39. Social Studies of Science, 19(3), 387420. https://doi.org/10.1177/030631289019003001
Star, S.L. y Ruhleder, K. (1996). Steps Toward an Ecology of Infrastructure: Design and Access for Large Information Spaces. Information Systems Research, 7(1), 111-134. https://doi.org/10.1287/isre.7.1.111

Sassen, S. (2005). Cities as Strategic Sites. Sociology, 39(2), 343-370. https://doi.org/10.1177/0038038505050544

Vergara Vidal, J. (2017). Verticalización: la edificación en altura en la Región Metropolitana de Santiago (19902014). INVI, 32(90), 9-49. http://dx.doi.org/10.4067/S071883582017000200009

Vergara Vidal, J. (2018). Edificaciones en la Región Metropolitana de Santiago (1990-2014). Sus estrategias materiales. Bitácora Urbano Territorial, 28(3), 9-18. https://doi.org/10.15446/bitacora.v28n3.59073

Vinck, D. (2009). De l'objet intermédiaire à l'objetfrontière: Vers la prise en compte du travail d'équipement. Revue d'anthropologie des connaissances, 3,1(1), 51-72. https://dx.doi.org/10.3917/rac.006.0051

Vinck, D. (2017). El papel de los objetos intermediadores en la interdisciplinariedad. PontodeAcesso, 11(1), 325. https://doi.org/10.9771/rpa.v11i1.23172

Zegers, Cazú (1998). La ética de los materiales. Ensayo sobre la condición matérica de la arquitectura. Arq, (39), 16-17. Disponible en http://www.edicionesarq.cl/1998/arq-39-materia/ 